\title{
Parity Effect and Charge Binding Transition in Submicron Josephson Junction Arrays
}

\author{
M. V. Feigel'man, S. E. Korshunov and A. B. Pugachev \\ L. D. Landau Institute for Theoretical Physics, Moscow 117940, RUSSIA
}

(October 9, 2018)

\begin{abstract}
We reconsider the issue of Berezinskii-Kosterlitz-Thouless (BKT) transition into an insulating state in the Coulomb-dominated Josephson junction arrays. We show that previously predicted picture of the Cooper-pair BKT transtion at $T=T_{2}$ is valid only under the condition that $T_{2}$ is considerably below the parity-effect temperature $T^{*} \approx 0.1 \Delta$ and even in this case it is not a rigorous phase transition but only a crossover, whereas the real phase transition takes place at $T_{1} \approx T_{2} / 4$. Our theory is in agreement with available experimental data on Coulomb-dominated Josephson arrays and also sheds some light on the origin of unusual reentrant temperature dependence of resistivity in the array with nearly-criticial ratio $E_{C} / E_{J}$.
\end{abstract}

1. Two-dimensional arrays of micron-scale superconducting islands are extensively studied during last years, both experimentally [1 1 and theoretically [5 mined by the competition between Josephson coupling energy $E_{J}$ and effective charging energy $E_{C}=e^{2} / 2 C$, where $C$ is some relevant electric capacitance (to be discussed below). Macroscopic superconductive coherence was observed at low tempratures in the arrays with $E_{J} \gg E_{C}$, whereas arrays with $E_{J} \ll E_{C}$ show insulating behaviour at $T \rightarrow 0$. At nearly-critical value of the ratio $x=E_{J} / E_{C} \sim x_{c r}$ direct transition between superconductive (SC) and insulating (I) behavior as function of $x$ was observed in zero magnetic field [2]. MOrevoer, very weak magnetic field $B \leq 1 G$ (producing small fractions of flux quantum per unit cell of the array) was shown to switch arrays with $x \approx x_{c r}$ between SC-like and I -like behavior as function of temperature; recently very interesting intermediate region was found [2] where resistance $R(T)$ is basically constant in the temperature range $10 m K \leq T \leq 200 m K$, which indicates the existence of a "2D metal" state sandwiched between SC and I phases.

The above-mentioned basic properties of 2D arrays are in qualitative agreement with available theories [5, ]7] (except for the recently observed 2D metal state); however several important features are not understood yet. In particular, resistance of the insulating arrays shows purely activated behaviour $R(T) \propto \exp \left(E_{a} / T\right)$ with constant activation energy $E_{a}$ through the whole temperature interval studied [2, 4 , whereas theoretically the charge binding BerezinskiiKosterlitz-Thouless (BKT) transition [8.90 from the conducting to insulating phase is expected to occur [5] at the temperature $T_{2} \approx E_{C} / \pi$. Such a transition should occur due to nearly-logarithmic form of Coulomb interaction between charges in the arrays with self-capacitance of islands $C_{0}$ very small compared to the inter-island (junction) capacitance $C$. In the currently studied arrays the ratio $C / C_{0} \sim 100$ (as measured at very low temperatures, about $10 \mathrm{mK}$, cf. e.g. [2]), which should result in the logarithmic interaction throughout the whole array (effecitve length of interaction $\Lambda$ should be estimated with the account of 3D nature of electric field, which leads [2]10] to $\Lambda \sim C / C_{0} \sim 100$ ) and, consequently, to the charge binding BKT transition.

In the case of islands in SC state and under the condition $E_{J} \ll E_{C}$ the temperature of this transtion was estimated [5] as $T_{2} \approx E_{C} / \pi$, whereas in the case of normal islands (i.e. with superconductivity suppressed by magnetic field) it is expected to be 4 times lower: $T_{1} \approx E_{C} / 4 \pi$ due to the twofold decrease of an elementary charge available. Nevertheless no indication of such a transtion in array of SC islands was found experimentally (except in very recent preprint [11], which is discussed below). Another surprising feature observed in [2] is nonmonotoneous ("reentrant") temperature behaviour of resistance $R(T)$ of the array with a nearly-critical $E_{J} / E_{C}$ ratio at $T \leq 200 \mathrm{mK}$.

In the present Letter we show that the above experimental observations can be naturally understood once the temperature dependence of effective Coulomb interation between the charges in the array is taken into account properly. In a very broad sense our analysis follows the ideas of Efetov [12 who established the background for the description of quantum fluctuations in granular superconductors; namely, we consider screening of Cooper-pair Coulomb interaction by normal quasiparticles existing in each superconductive island at finite temperatures. However we believe that Efetov's treatment of the effect he proposed technically was not quite correct, thus we present here another theoretical approach to the same problem.

Our main qualitative result can be formulated as follows: at the temperatures above the so-called parity-effect [13 16] temperature $T^{*} \approx \Delta / \ln \mathcal{M} \ll \Delta$ [where $\mathcal{M}=V \nu(0) \sqrt{8 \pi T \Delta} \sim 10^{4}-10^{5}, V$ is the volume of the island and $\nu(0)$ is the density of states at the Fermi level in absense of superconductivity] the presence of thermal quasiparticles [with the number $\sim \mathcal{M} \exp (-\Delta / T) \gg 1$ ] on each island excludes any possibility to observe Cooper-pairs BKT transtion at $T_{2}$. Since in the most of arrays studied till now the above-defined characterisitic temperature $T_{2}$ was in the range 
0.3-0.5 K, whereas parity effect temperature $T^{*} \approx 0.2 K<T_{2}$, the absense of anything like BKT transtion near $T_{2}$ is quite natural (measurements below $T^{*}$ were not possible in these arrays [3, 3 ] since $R(T)$ becomes immeasurably high $\left.\left[\geq 10^{9} \mathrm{Ohm}\right]\right)$. On the other hand single-electron BKT transtion is a completely different issue: we do expect such a transition to be observable at approximately the same temperature $T_{1} \approx T_{2} / 4$ as in the arrays with islands in the normal state.

2. We proceed now to the derivation of our results, and will follow, with one important modification, Ref. [5]. In the limit when charge tunneling is weak and is important only for the establishment of thermodynamic equilibrium an array of superconducting islands can be described by a classical partition function of a form:

$$
Z=\sum_{\{n\}} \exp \left[-\frac{1}{2} \sum_{i, j} G_{i j} n_{i} n_{j}-\frac{D}{T} \sum_{j} \frac{1-(-1)^{n_{j}}}{2}\right] ; \quad G_{i j}=\frac{e^{2}}{T} C_{i j}^{-1}
$$

The first term in the exponent in Eq. (1) stands for the electrostatic energy of the array which in the case when only mutual capacitance of nearest islands $C$ is of importance corresponds to the logarithmic interaction of the charges in two-dimensional array:

$$
G_{i=j}-G_{i j} \approx \frac{2 E_{C}}{T}\left(\frac{1}{2 \pi} \ln R_{i j}+\frac{1}{4}\right) ; \quad E_{C}=\frac{e^{2}}{2 C}
$$

whereas the second term describes the dependence of a free energy of a superconducting island on the parity of the number of electrons $n_{j}$ on this island [13, 15, 16].

The free energy difference $D(T)$ between the islands with odd and even number of electrons can be expressed as

$$
D(T)=-T \ln \tanh \left(\Omega_{o e} / T\right)
$$

where $\Omega_{o e}=-T \ln \left(Z_{o d d} / Z\right)$ and $Z_{o d d}$ is the "odd grand canonical partition function" introduced in Ref. [13] for the study of parity effect. Accurate expression for the function $\Omega_{o e}(T)$ can be found in $[15]$, but for $T \ll \Delta$ a good approximation is given by

$$
\Omega_{o e}(T) / T \approx \mathcal{M} e^{-\Delta / T} ; \quad \mathcal{M}=V \nu(0) \sqrt{8 \pi T \Delta}
$$

The ratio $\Omega_{o e} / T$ in that limit is proportional to the number of thermally exited quasiparticles on one island.

In terms of statistical mechanics partition function (1) defines a lattice Coulomb gas in which the fugacities of odd charges $Y=\exp (-D / T)$ differ from the fugacities of even charges (which are equal to one). Comparison of Eq. (4) with Eq. (3) shows then that for $T \ll T^{*}=\Delta / \ln \mathcal{M}$ the parity-dependent free energy difference $D(T) \approx \Delta-T \ln \mathcal{M} \gg T$ and $Y \ll 1$, whereas in the opposite limit $T \gg T^{*}$ the quantity $D(T)$ becomes exponentially small and $Y$ is very close to one.

Previously it has been assumed [5] that in the regime when island charges behave as classical variables the main difference between the array of normal islands and the array of superconducting islands is that in the array of normal islands the charge of each island is quantized in units of $e$, whereas in the array of superconducting islands the charge is quantized in units of $2 e$. The consequence for the array the electrostatic properties of which are dominated by mutual capacitance of nearest neighbours is that the temperature $T_{2}$ of the BKT transition in the array of superconducting islands (appearance of free double charges) should be exactly four times higher than the temperature $T_{1} \sim E_{C} / 4 \pi$ of the BKT transition in the analogous array of normal islands (appearance of free single charges). Comparison with Eq. (1) shows that such description of the array of superconducting islands would be correct only in the limit of $D(T) / T \rightarrow \infty$. Since $D(T)$ is always finite this description turns out to be misleading. The behaviour of the array at temperatures close to $T_{2}=E_{C} / \pi$ depends qualitatively on the relation between $T_{2}$ and $T^{*}$; we consider both cases in turn.

3. At $T_{2} \geq T^{*}$ an array of the superconducting islands is described by practically the same partition function as an array of the normal islands, since $D\left(T_{2}\right)<<T_{2}$ in that case. The phase transition into insulating state in such system can be associated with the binding of the charges \pm 1 into neutral pairs; it takes place at the temperature $T_{1}$ which is slightly lower than the simple estimate $T_{1}^{(0)}=E_{C} / 4 \pi$ which can be obtained by comparison of the single charge energy with its entropy. The difference between $T_{1}$ and $T_{1}^{(0)}$ is related to the renormalization of charge interaction by bound pairs of charges and decreases with decrease in fugacities. The appearance of the free single charges induces the screening of the Coulomb interaction for all types of charges and therefore the double charges also are free at $T>T_{1}$. Not even a trace of a separate phase transition related to debounding of double charges can be expected to be observed in such a situation, which was realized in the experiments [1] 4 . 
4. In the opposite case $T_{2}<T^{*}$ there is a range of temperatures $T_{2}<T<T^{*}$ where fugacity of single charges $Y$ is much smaller than one. This leads to the increase of phase transition temperature, but it still has to remain smaller than $T_{1}^{(0)}$. The difference with the case of $Y \approx 1$ is that for $Y \ll 1$ the concentration of free single charges $n_{1}$ remains small even at the temperatures considerably higher than $T_{1}$. In the region $T_{1}<T<T_{2}$ it can be estimated with the use of the standard Debye-Hückel approximation which gives for $n_{1}$ the self-consistent equation:

$$
n_{1}=2 Y \exp \left\{-\frac{1}{2} \int \frac{d^{2} \mathbf{q}}{(2 \pi)^{2}} \frac{1}{K\left[2\left(1-\cos q_{x}\right)+2\left(1-\cos q_{y}\right)\right]+n_{1}}\right\}
$$

(where $K=T / 2 E_{C}$ ) the solution of which for small $n_{1}$ can be expressed as

$$
n_{1} \approx 2 \exp \left\{-\frac{D(T)+a E_{C}-\left[\ln \left(4 E_{C} / T\right)\right] E_{C} / 4 \pi}{T-T_{1}^{(0)}}\right\}
$$

where $a=0.276 \ldots$. The main effect of Coulomb interaction is seen in that it produces singularity at $T=T_{1}^{(0)}$ in the exponent in Eq.(6). If the shift of the phase transition temperature is taken into acccount $T_{1}^{(0)}$ should be substituted by $T_{1}$. Note that second and third terms in the numenator of the exponent almost canceal each other in the relevant range of parameters.

For $D(T) \gg T$ the screening of the interaction is noticable only on the large scales and the concentration of free double charges also remains small. On the other hand at the temperature $T_{2}=4 T_{1} \approx E_{C} / \pi$ the free double charges have to appear even when $Y=0$ due to mutual influence of pairs of double charges (cf. with Ref. [5]). That means that for $D(T) \gg T$ in the vicinity of $T_{2}$ there occurs a crossover characterized by a prolifiration of free double charges.

Close to the transition temperature $T_{1}$ the self-consistent approximation is no longer valid and more advanced methods should be used. It is easy to show that when only the single and double charges are taken into account, the Coulomb gas model described by partition function (1) becomes isomorphic (in continuous approximation) to the sine-Gordon model defined by the Hamiltonian:

$$
H=\int d^{2} \mathbf{r}\left[\frac{K}{2}(\nabla \theta)^{2}-2 Y \cos \theta-2 \cos 2 \theta\right] ; \quad K=\frac{T}{2 E_{C}}
$$

The renormalization group equations for the Hamiltonian (7) can be found in Ref. [17]. As can be expected their solution shows that for temperatures lower than $T_{2}$ the presence of double charges (in form of neutral pairs) does not introduce any qualitative changes. In the close vicinity of $T_{1}$ the temperature dependence of $n_{1}$ deviates from the self-consistent result (6) and follows the standard BKT critical behaviour [9] with

$$
n_{1}(T) \propto \exp \left[-\frac{b}{\sqrt{1-T / T_{1}}}\right]
$$

where $b$ is of the order of unity. The array's linear dc resistance should be inversely proportional to the density of free charges $n_{1}$. Taking into account Eqs.(6, 8), we get an estimate for this resistance at the temperatures near $T_{1}$ :

$$
\ln \frac{R(T)}{R_{1}} \approx \min \left[\frac{D(T)}{T-T_{1}}, \frac{b}{\sqrt{4 \pi}}\left(\frac{E_{C}}{T-T_{1}}\right)^{1 / 2}\right]
$$

where $R_{1}$ is inversely proportional to the probability of tunnelling event which is only weakly dependent on the temperature: $R_{1} \sim R_{n} \mathcal{M}$, where $R_{n}$ is the normal-state tunnelling resistance (cf. [18]).

5. The representation (耳) is also useful for comparison between our results and Efetov's treatment of screening by quasiparticles [12], which can be expressed just as the replacement of the original capacitance matrix $C_{i j}$ by the "effective" one defined (at $T \ll \Delta$ ) as

$$
C_{i j}^{e f f}=C_{i j}+\delta_{i j} \cdot V \nu(0)(2 e)^{2} \sqrt{\frac{2 \pi \Delta}{T}} e^{-\Delta / T}
$$

Let us now formally expand the second term in Eq.(7) up to the second order in $\theta$, and neglect the rest of terms. One can easily see that the expression obtained in this way for the effective interaction between 2e charges (generated by expansion of the partition function in powers of the $\cos 2 \theta$ term) would coinside with the one obtained by inversion 
of the effective capacitance (10). Physically the above formal operation would mean neglect of the discrete nature of electric charge, which could be reasonable if charge transport between islands could proceed via some "classical" channels able to provide charge in continuous amounts (like charging of macroscopic electric capacitor by external voltage source). It is not the case for submicron arrays with tunnel junctions where charges of islands can change only

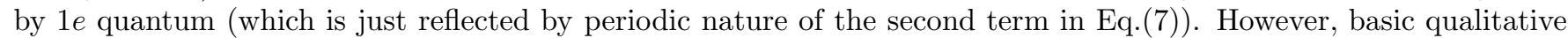
feature of Efetov's result - screening of Cooper pair charges by normal exitations - remanins valid, in spite of the absense of any simple notion like an effective capacitance matrix.

6. It follows from our results, that in order to observe some growth of effective activation energy [defined as $\left.E_{a}=d \ln R(T) / d(1 / T)\right]$ with $T$ approaching $T_{2}$ from above, one needs to use array with the Coulomb energy $E_{C}$ few times below than $\Delta$, so that $T_{2} \leq T^{*}$. This conclusion is in complete agreement with recent experimental data [11], where some moderate growth of $E_{a}(T)$ in the temperature range around 0.2-0.3 K was observed. This experiment differs from few previous ones of the same type [2 1 (where constant $E_{a}$ was observed) by lower values of $E_{C}$ and a bit higher reported $\Delta$. On the other hand, we do not agree with the interpretation of that $E_{a}(T)$ growth as precursor of the BKT transtion at $T_{2}$, given in [11] for their SC arrays. As follows from our results, no such a transtion exists at $T_{2}$, which agrees with a rather modest (compared to BKT behaviour) growth of $E_{a}(T)$ observed in 11 at $T \sim T_{2}$. Note that the agreeement between the data reported in [11] for normal arrays and the expected genuine BKT transition at $T_{1}$ is much better than the above-mentioned comparison for SC arrays.

The above theoretical results point out that any analysis of experimental data on superconductor-insulator transtion in artificial arrays of superconducting islands (as well as in the dirty thin films near SC-I transtion) should take into account an existence of a characteristic temperature scale of the parity effect $T^{*}$ (note that $T^{*}$ is magnetic-field dependent and strongly suppressed by the fields of the order of $H_{c 2}$ ). In particular, the behavior of I-V characteristics in the intermediate temperature range $T^{*}<T<\Delta$ cannot be unambigously related to the genuine ground-state properties of the system, as is examplified by non-monotonous $R(T)$ behaviour observed in [2] for a nearly critical ratio $E_{J} / E_{C}$. We interpret this unusual behaviour as follows: at moderately low $T$ screening by quasiparticles is effective and reduces Coulomb repulsion of Cooper pairs, leading to the decrease of $R(T)$ behaviour; at still lower $T$ this screening is gone, Coulomb repulsion increases and effective ratio $E_{J} / E_{C}^{e f f}$ enters the "insulative" part of the phase diagram, leading to the increase of $R(T)$ at further $T$ decrease.

We are grateful to V. B. Geshkenbein, A. Kitaev, N. B. Kopnin, A. I. Larkin, J. E. Mooji, Yu. V. Nazarov, G. Schön, M.Skvortsov and H. van der Zant for many helpful discussions. Financial support from INTAS-RFBR grant \# 95-0302 (M.V.F.) and Swiss National Science Foundation collaboration grant \# 7SUP J048531 (M.V.F. and A.V.P.), as well as the DGA grant \# 94-1189 (M.V.F.) and RFBR grant \# 96-02-18985 (S.E.K) is gratefully acknowledged.

[1] H.S.J. van der Zant, F.C.Fritschy, W.J.Elion, L.J.Geerlings and J.E.Mooji, Phys. Rev. Lett. 69, 2971 (1992).

[2] H.S.J. van der Zant, W.J.Elion, L.J.Geerlings and J.E.Mooji, Phys. Rev. B 54, 10081 (1996).

[3] T.S.Tighe, M.T.Tuominen, J.M.Hergenrother and M.Tinkham, Phys. Rev. B 47, 1145 (1993).

[4] P.Delsing, C.D.Chen, D.B.Haviland, Y.Harada and T.Claeson, Phys. Rev. B 50, 3959 (1994).

[5] R. Fazio and G. Schön, Phys.Rev. B 43, 5307 (1991).

[6] R.Fazio et al, Helv.Phys.Acta 65, 228 (1992).

[7] Proceedings of the NATO Advanced Research Workshop on Mesoscopic Superconductivity, Karlsruhe 1994, Eds. F.Hekking, G.Schoen and D.Averin [Physica B 203, issues 3-4 (1994)].

[8] V.L.Berezinskii, ZhETF 59, 907 (1970) [Sov.Phys. - JETP 32, 493 (1971)]; J. M. Kosterlitz and D. J. Thouless, J.Phys.C6, $1181(1973)$.

[9] J.M. Kosterlitz J.Phys.C7, 1046 (1974).

[10] J.E.Mooji and G.Schoen, in Single Electron Tunnelling, eds. H.Grabert and M.Devoret (Plenum, New York 1992), Chapter 8 and references therein.

[11] A.Kanda and S.Kobayashi, preprint, submitted to Phys. Rev. B. (1996).

[12] K.B.Efetov, ZhETF 78, 2017 (1980) [Sov.Phys. - JETP 51, 1015 (1980)].

[13] M.T.Tuominen, J.M.Hergenrother, T.S.Tighe and M.Tinkham, Phys. Rev. Lett. 69,1997 (1992).

[14] F.W.J.Hekking et al, Phys. Rev. Lett.70, 4138 (1993); P.Joyez et al, Phys. Rev. Lett. 72, 2458 (1994).

[15] D. V. Averin and Yu. V. Nazarov, in [f], p.310.

[16] L. Glazman et al, in [7], p.316.

[17] J. A. Jaszczak and W. F. Saam, Phys. Rev. B 37, 7619 (1988).

[18] B. Janko and V. Ambegaokar, Phys. Rev. Lett. 75, 1154 (1995). 